\title{
Anodal Transcranial Direct-Current Stimulation Over the Right Dorsolateral Prefrontal Cortex Influences Emotional Face Perception
}

\author{
Li-Chuan Yang ${ }^{1,3} \cdot$ Ping $\operatorname{Ren}^{1,4} \cdot$ Yuan-Ye Ma ${ }^{1,2}$
}

Received: 28 November 2017/ Accepted: 16 April 2018/Published online: 14 June 2018

(C) The Author(s) 2018

\begin{abstract}
The dorsolateral prefrontal cortex (DLPFC) is considered to play a crucial role in many high-level functions, such as cognitive control and emotional regulation. Many studies have reported that the DLPFC can be activated during the processing of emotional information in tasks requiring working memory. However, it is still not clear whether modulating the activity of the DLPFC influences emotional perception in a detection task. In the present study, using transcranial direct-current stimulation (tDCS), we investigated (1) whether modulating the right DLPFC influences emotional face processing in a detection task, and (2) whether the DLPFC plays equal roles in processing positive and negative emotional faces. The results showed that anodal tDCS over the right DLPFC specifically facilitated the perception of positive faces, but did not influence the processing of negative faces. In addition, anodal tDCS over the right primary visual cortex enhanced performance in the detection task regardless of emotional valence. Our findings suggest, for the first time,
\end{abstract}

Yuan-Ye Ma

yuanma0716@vip.sina.com

Ping Ren

greenrpg@hotmail.com

1 Laboratory of Brain and Cognition, Kunming Institute of Zoology, Chinese Academy of Sciences, Kunming 650223, China

2 State Key Laboratory of Brain and Cognition, Institute of Biophysics, Chinese Academy of Sciences, Beijing 100101, China

3 University of the Chinese Academy of Sciences, Beijing 100049, China

4 School of Nursing, University of Rochester Medical Center, Rochester, NY 14642, USA that modulating the right DLPFC influences emotional face perception, especially faces showing positive emotion.

Keywords tDCS · Facial expression - Emotion · Dorsolateral prefrontal cortex

\section{Introduction}

The dorsolateral prefrontal cortex (DLPFC) is considered to play a crucial role in many high-level functions, such as working memory, decision-making, and self-control [1-4]. In addition, increasing numbers of studies have demonstrated that the DLPFC is also involved in normal and abnormal emotion-related brain networks, along with the amygdala and other subcortical structures [5, 6]. Pleasant words induce more activity in the bilateral DLPFC than unpleasant words [7]. And the DLPFC is activated by pleasant visual stimuli and reduced by unpleasant ones in a working memory task [8]. In an electroencephalographic (EEG) study, Godinho et al. found that the right DLPFC is involved in the emotional modulation of pain [9]. In addition, some studies have also found that the DLPFC is involved in cognitive tasks related to emotional faces $[10,11]$.

Even though many studies have shown that the DLPFC is important for emotional modulation and emotional memory, the contribution of each hemisphere is controversial. One theory is that the right hemisphere is predominantly involved in processing emotion, and the left hemisphere is specialized for cognitive processes $[12,13]$. In a working memory-related functional magnetic resonance imaging (fMRI) study, although the left DLPFC showed a pattern of activity similar to the right DLPFC, the general pattern is consistent with a right-lateralization of 
face and emotional distracters [14]. A previous study has reported that patients undergoing antidepressant treatment for major depression showed significantly increased right DLPFC activity in response to unattended fear-related stimuli [15]. Another study found that patients with depression exhibit diminished right DLPFC activation and reduced right DLPFC-amygdala coupling during emotion regulation [16]. Most of the previous studies have shown that the DLPFC, especially the right DLPFC, is involved in emotion-related working memory tasks, but it is still not clear whether the DLPFC directly modulates the detection of emotional facial expressions.

Transcranial direct-current stimulation (tDCS) is a noninvasive technique that is widely used to treat many diseases and to study cognitive processes. Anodal tDCS is considered to facilitate brain activity, whereas cathodal stimulation suppresses it. Several studies have demonstrated that anodal tDCS over the DLPFC significantly improves the emotional state of patients suffering emotional disorders, such as depression and autism [17-19]. In addition, several studies have shown that anodal stimulation over the DLPFC improves probabilistic classification learning [20], declarative memory [21], working memory [22], driving behavior [23], and decision-making [24, 25], while diminishing risk-taking behavior [26]. Recently, Penolazzi et al. reported a significant effect of tDCS on emotional memory: right anode/left cathode selectively facilitates the memory of pleasant images whereas left anode/right cathode selectively facilitates the memory of unpleasant ones [27]. So far as we know, there is no evidence that the right DLPFC directly modulates the detection of emotional facial expression.

In the present study, we set out to investigate the role of the right DLPFC in emotional face processing using tDCS. Our aims were to investigate whether emotional face detection can be modulated by stimulating the right DLPFC and to test the right-hemisphere hypothesis. Since some previous studies have reported an after-effect of tDCS in emotion regulation $[28,29]$, we also examined this after-effect.

\section{Materials and Methods}

\section{Participants}

All participants were right-handed with normal or corrected-to-normal vision. Seventy-eight undergraduates were paid to participate in four experiments. The participants in each experiment were as follows: Experiment 1 (12 females and 11 males; mean age, 23 years, range 19-29), Experiment 2 (13 females and 4 males; 21 years, 19-24), Experiment 3 (10 females and 10 males; 25 years, 21-29), and Experiment 4 (9 females and 9 males; 22 years, 19-24). All participants were blind to the purpose of the experiments. Each participant was given instructions for the experiment and provided written informed consent in accordance with procedures approved by the Ethics Committee of the Kunming Institute of Zoology, Chinese Academy of Sciences. All experiments were conducted in accordance with the ethical standards of the 1964 Declaration of Helsinki.

\section{Apparatus and Stimuli}

All experiments were conducted in a dimly-lit soundattenuated room. Participants were seated $70 \mathrm{~cm}$ in front of a display and kept their head on the chin-rest during the entire experiment. Visual stimuli were presented on a 19-inch CRT display. The screen resolution was $1024 \times 768$, and the refresh rate was $100 \mathrm{~Hz}$. Participants responded using a keyboard. The experimental programs were written with MATLAB 6.5 and Psychtoolbox 2.54, which were supplied by Institute of Biophysics, Beijing, China.

The visual stimuli contained four schematic faces (Fig. 1A) with two different conditions: one sad and three neutral faces, and one happy and three neutral faces. The visual angle of the image was $5.4^{\circ}$, and each schematic face was $2.5^{\circ}$ with a $0.4^{\circ}$ gap between them. In Experiment 4 , the visual stimuli were replaced by scrambled faces (Fig. 1B). There were 6 different contrast levels of visual stimuli (relative to background): $1.1 \%, 2.2 \%, 3.3 \%, 4.4 \%$, $5.6 \%$, and $6.7 \%$.

\section{Transcranial Direct-Current Stimulation}

tDCS was delivered by a home-made battery-driven constant-current stimulator, at the State Key Lab of Brain and Cognitive Sciences, Institute of Biophysics, Beijing, China. The current was $1.5 \mathrm{~mA}$ and induced through two salinesoaked sponge electrodes $\left(30 \mathrm{~cm}^{2}\right.$ each). Previous studies have shown that a current intensity of no more than $2 \mathrm{~mA}$ is safe in normal participants. To locate the right DLPFC, the EEG 10/20 system is sufficient when using traditional tDCS electrodes, and has been widely used in many studies [30, 31]. In Experiments 1, 2, and 4, to stimulate the right DLPFC, the anode was placed over F4 (according to the EEG $10 / 20$ system) and the cathode was over the supraorbital region. In Experiment 3, the anode was applied over O2 to stimulate the right primary visual cortex.

\section{Procedure}

The stimulus sequence is illustrated in Fig. 1C. Each trial began with a "Ready?" placed in the center of the screen, and participants pressed the space-bar to start. After $500 \mathrm{~ms}$ fixation, the emotional faces were displayed for $30 \mathrm{~ms}$, and 
Fig. 1 Emotional face detection task. A In Experiments 1, 2 and 3 , the visual stimulus contained four schematic faces (one emotional and three neutral).

B In Experiment 4, the visual stimulus was replaced by four scrambled faces. C The sequence of stimuli in the experimental procedure.
A
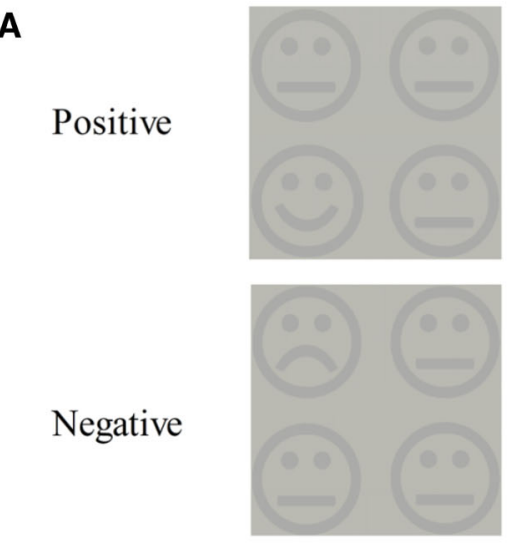

B

Target 1

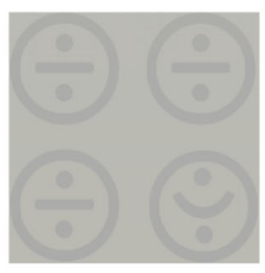

Target 2

C

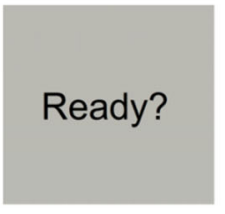

Start

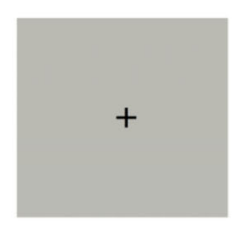

$500 \mathrm{~ms}$

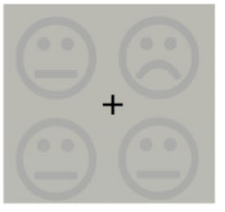

$30 \mathrm{~ms}$

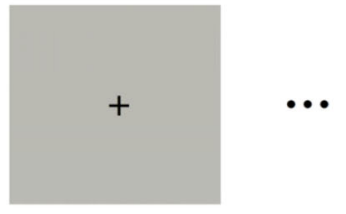

Judge then replaced by a blank screen. The participants were asked to press ' 5 ' on the keypad if a sad face was displayed, and ' 6 ' if a happy face was displayed. Before the formal test, there was a short practice with 10-20 trials to help participants become familiar with the task. In Experiment 1, there were 2 formal test sessions, Session 1 without tDCS and Session 2 with tDCS, which were designed to examine the direct effect of tDCS. Each session consisted of 240 trials, including 20 for each emotional face under each contrast level. There was a short rest ( $\sim 10 \mathrm{~min}$ ) between the two sessions. At the beginning of Session 2, the direct current was manually ramped up to $1.5 \mathrm{~mA}$ in $30 \mathrm{~s}$. After $3 \mathrm{~min}$ adaptation to the stimulation, participants completed the same task as in Session 1. The experimental design was 2 (before and during tDCS) $\times 2$ (sad and happy faces) $\times 6$ (six contrast levels). Experiment 2 was designed to examine the after-effects of tDCS, and was essentially the same as Experiment 1 except for the following: after Session 1 in Experiment 2, the participants received tDCS stimulation for 15 min without a task; then Session 2 started immediately after cutting off the current, keeping the electrodes on the scalp until the rest of the experiment was finished. There were 336 trials in each part, with 28 trials in each condition. For comparison with the right DLPFC, the procedure of Experiment 3 was exactly the same as Experiment 1, except that the anodal electrode was moved to the right primary visual cortex $(\mathrm{O} 2)$. In Experiment 4, all the schematic faces were scrambled to disrupt emotional information, and compared with the other three emotion-related experiments (Fig. 1B). The electrode locations and experimental design were exactly the same as in Experiment 1.

\section{Results}

\section{Experiment 1}

Incorrect responses were excluded before analysis. Repeated measures analyses of variances (ANOVAs) were used to analyze the accuracy data. A Greenhouse-Geisser correction was used to correct for unequal variances. The alpha level was 0.05 for all data analyses. There was a main effect of contrast level whereby better performance occurred with higher contrast levels $[F(5,110)=$ 117.636, $P<0.001]$. Also, the interaction between tDCS and facial expression was significant $[F(1,22)=7.448, P=$ 0.012; Fig. 2A]. After ANOVAs, paired $t$-tests were used to determine the significance details in each condition. Post-hoc $t$-tests showed that the accuracy of detecting a happy face was significantly higher during $(74.75 \%)$ than before $(70.58 \%)$ anodal tDCS $(P=0.036)$. However, the response to a sad face was not influenced by tDCS (before: $70.58 \%$, during: $70.73 \%)(P=0.569)$. During tDCS, the perception rate of the positive face was higher $(74.75 \%)$ than that of the negative face $(70.73 \%)(P=0.029)$. Moreover, the performance was significantly better for a positive face during $(88.48 \%)$ than before $(82.39 \%)$ tDCS 
Fig. 2 Performance in Experiment 1. A Performance was significantly better for a positive but not for a negative face, with the anode over the right DLPFC (dashed line, chance level of performance). B Average accuracy rates at each contrast level for positive and negative faces. $\left({ }^{*} P<0.05 ;{ }^{\#} P<0.1\right)$.

\section{A Anodal tDCS over the right DLPFC}

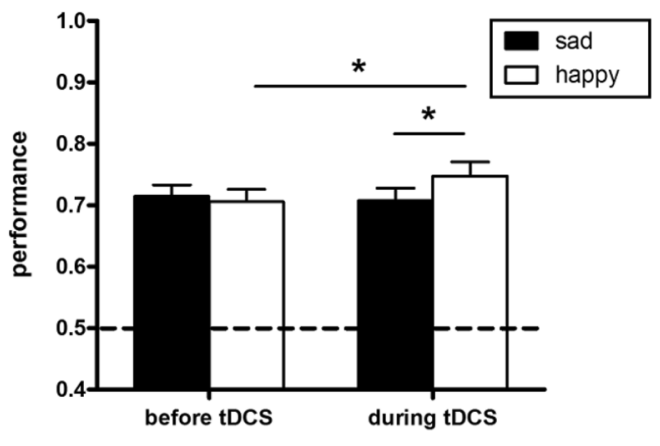

B

Anodal tDCS: happy faces

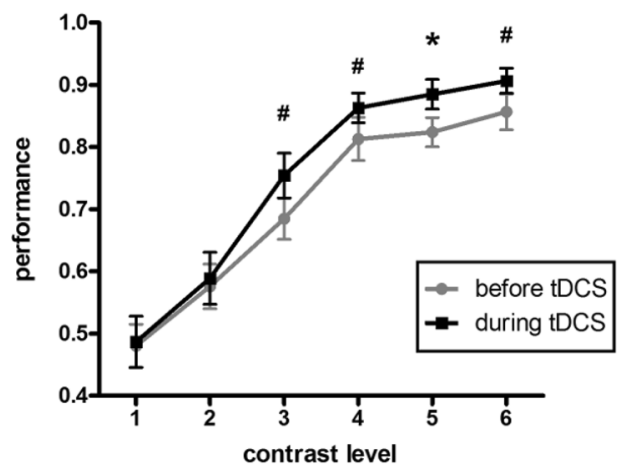

Anodal tDCS: sad faces

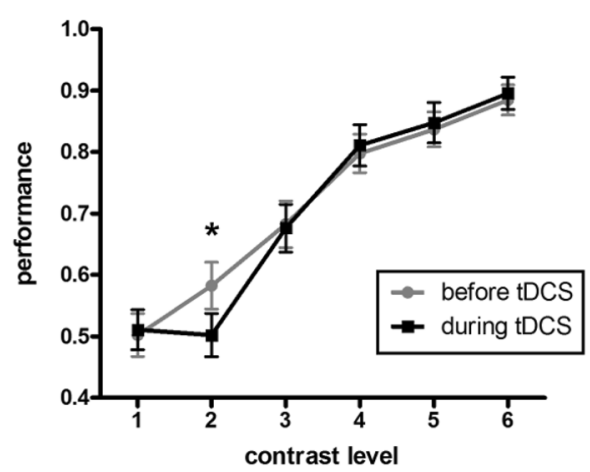

at contrast level $5(P=0.011)$. Similarly, the responses were slightly but not significantly better for a positive face during $(75.43 \%, 86.30 \%$, and $90.65 \%)$ than before $(68.48 \%, 81.30 \%$, and $85.65 \%)$ tDCS at contrast levels 3 $(P=0.071), 4(P=0.061)$, and $6(P=0.099)$ (Fig. 2B). For a negative face, the responses were significantly worse during $(50.22 \%)$ than before $(58.26 \%)$ tDCS only at contrast level $2(P=0.009)$.

\section{Experiment 2}

The data analysis was the same as in Experiment 1. There was a main effect of contrast level, with better performance at higher levels $[F(5,80)=114.037, P<0.001]$. The interaction between tDCS and facial expression was not significant $[F(1,16)=1.621, P=0.221]$ (Fig. 3A).

\section{Experiment 3}

There was a main effect of contrast level, with better performance at higher levels $[F(5,95)=119.848, P<$ 0.001]. The main effect of tDCS showed that anodal tDCS over O2 slightly enhanced the accuracy (before: $73.15 \%$, during: $74.69 \%)[F(1,19)=4.263, P=0.053]$. The interaction between tDCS and facial expression was not significant $[F(1,19)<1$; Fig. 3B].

\section{Experiment 4}

There was a main effect of contrast level with better performance at higher levels $[F(5,85)=126.804, P<$ 0.001]. There was a main effect of tDCS, with better responses during $(78.06 \%)$ than before tDCS $(75.83 \%)$ $[F(1,17)=4.761, P=0.043]$. The interaction between tDCS and facial expression was not significant $[\mathrm{F}(1,17)=$ 2.566, $P=0.128$; Fig. 3C].

\section{Discussion}

In the current study, our results showed that tDCS is an effective and reliable tool to modulate brain activity. We found that anodal tDCS over the right DLPFC selectively facilitated the perception of positive but not negative faces. The effect of direct current only occurred during stimulation, and disappeared after removal of tDCS. The anodal stimulation over the right visual cortex also induced a slight enhancement of task performance with no difference between positive and negative faces. To the best of our knowledge, this is the first evidence of a relationship between the right DLPFC and emotional face detection using the tDCS technique.

In Experiment 1, using a two-forced-choice task, we investigated whether stimulating the right DLPFC could 
Fig. 3 Performance in Experiments 2-4. A With the anode over the right DLPFC, there was no significant influence on emotional face perception after removing tDCS. B With the anode over the right primary visual cortex, there was only a slight tDCS effect on emotional face perception, with no difference between positive and negative faces. $\mathbf{C}$ Performance in the non-face task was significantly better when the anode was over the right DLPFC. $(* P<0.05)$.
A Anodal tDCS over the right DLPFC: after effect

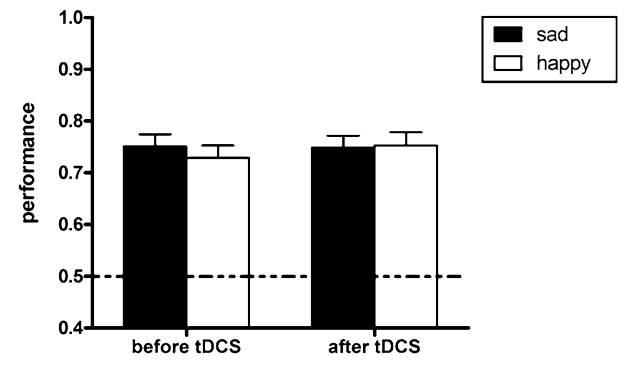

B Anodal tDCs over the right visual cortex

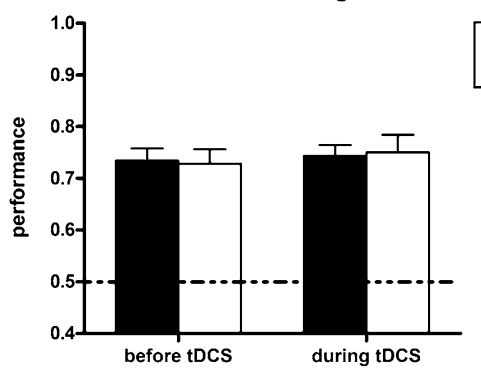

C Anodal tDCs over the right: scrambled faces

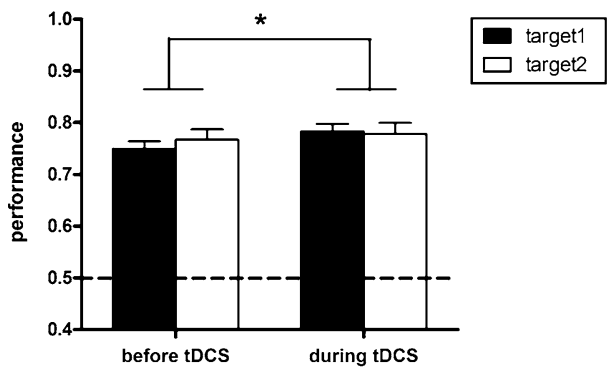

influence emotional face perception. Some previous tDCS studies have shown that the DLPFC plays an important role in the cognitive processes modulated by emotion [27] and several mental diseases (such as depression and pain) [18, 28, 29, 32]. In an fMRI study, Perlstein and colleagues showed that the right DLPFC is influenced by emotional pictures only in a working memory task but not in a detection task [8]. However, we found that the right DLPFC directly modulated performance in the emotional face detection task, which indicates that the DLPFC plays a more general role in processing emotional information regardless of the task. Different from the hypothesis of right hemisphere-emotion and left hemisphere-cognition, there is a valence-lateralization hypothesis which assumes that the right hemisphere is dominant for negative emotions and the left hemisphere is dominant for positive emotions $[12,33,34]$. Our results partly support the valencelateralization hypothesis, except that the right DLPFC detects positive emotion. In line with our findings, Penolazzi et al. found that right anodal/left cathodal tDCS facilitates the recall of pleasant stimuli, whereas reversing the electrode polarity facilitates the recall of unpleasant stimuli [27]. fMRI studies have also reported that positive/pleasant stimuli promote more activity in the right DLPFC than negative/unpleasant stimuli [7, 8]. Since tDCS over the left DLPFC was not examined in the current study, it is difficult to speculate on hemispheric differences from our experimental design. In future studies, tDCS can be applied over the left and right DLPFC to compare the different effects in emotion detection. Compared with the anodal tDCS effect, cathodal tDCS is usually used to suppress brain activity. Although several studies have reported that the cathodal effects are not consistent and effective $[22,35,36]$, it is worth examining the difference between cathodal and anodal effects on the DLPFC.

In Experiment 2, we tested the after-effect of tDCS using exactly the same stimuli as in Experiment 1 . Some studies have reported that the effect of current over the DLPFC on working memory tasks can last several minutes to several hours after removing the tDCS [37, 38]. Improvement of emotion-related disorders has been found after anodal tDCS over the DLPFC [18]. However, we did not find a significant tDCS after-effect. Differing from those studies, we examined emotional face perception in the DLPFC after anodal tDCS, so the tDCS after-effect may be task-dependent. In addition, some may argue whether the tDCS effect is induced by the anodal electrode over the specific position (right DLPFC), or the cathode over the left supraorbital area. To clarify this problem, we moved the anode over the right primary visual cortex $(\mathrm{O} 2)$ and kept the cathode over the left supraorbital region in Experiment 3. Compared with Experiment 1, the results showed a slight enhancement for both positive and negative faces. This demonstrated that a specific facilitation of positive faces was caused by stimulating the right DLPFC. And our result is congruent with Jolij's finding that an emotional face task is disrupted by applying transcranial magnetic stimulation (TMS) to the visual cortex [39]. It is noteworthy that both emotion and localization tasks were disrupted by applying TMS to the visual cortex in Jolij's study, indicating that the visual cortex is not specific to emotion tasks. To be consistent 
with Experiment 1, the stimuli in Experiment 3 were still displayed at the center of the screen, which is not the optimal location for activating the right primary visual cortex. Therefore, we would expect a stronger tDCS effect if the stimuli were displayed in the left visual field. Both Experiments 2 and 3 indicated that the selective facilitation of positive face perception is due to direct anodal tDCS over the right DLPFC.

Since we used schematic rather than natural faces, it may be argued that tDCS facilitated not emotional information processing but local features, such as mouth curvature. To exclude this possibility, we disrupted the configural information of faces by scrambling them in Experiment 4. In this way, the differences between target and distractors were only due to local features (such as mouth curvature). Our results showed no interaction of tDCS and targets, which means that the different effects of tDCS on positive and negative faces are based on emotional information but not local features. Interestingly, we found marginal significance of the tDCS main effect, which may be due to the possibility that the DLPFC plays an important role in visual attention as well, which has been reported in some human and animal studies [40, 41].

Finally, we need to acknowledge several limitations in the current study. First, we applied the cathode over the left supraorbital cortex, which is close to the orbitofrontal cortex. Comparing the right DLPFC and the visual cortex, the results demonstrated that the tDCS effect on detecting emotional faces was dependent on the anode over the right DLPFC. Nevertheless, the location of the cathodal electrode may influence tDCS as well. Using several electrode combinations, Antal et al. found that only the occipitalvertex $(\mathrm{Oz}-\mathrm{Cz})$ electrode position elicited a tDCS effect, implying that the direction of current flow should be considered [42]. Some previous studies have also shown that the orbitofrontal cortex is involved in emotion-related processes $[8,43]$. In further studies, the cathode could be placed over other brain areas (such as $\mathrm{Cz}$ ) to determine whether a change of current direction influences the effects. Second, although Experiments 2 and 4 can be used to confirm the direct tDCS effect on the right DLPFC, there was no traditional sham stimulation in the present study. To better control for the placebo effect, sham experiments should be considered in future research. Besides, there were more female participants in Experiment 2. Although several tDCS studies have reported that males and females do not differ in responding to stimulation in the frontal lobe $[44,45]$, a gender effect could be a potential factor in assessing the effects of tDCS in emotion-related tasks.

tDCS is an effective and reliable non-invasive technique for modulating brain function. We found that direct anodal tDCS over the right DLPFC selectively facilitated positive face processing compared with negative face processing.
The results showed, for the first time, that modulating the DLPFC influences emotional face detection. In addition, the results support the hypothesis that the right hemisphere plays different roles in processing emotional information with different valences.

Acknowledgements This work was supported by the National Natural Science Foundation of China $(91132302,31730039)$ and Ministry of Science and Technology of China (2015CB351701).

\section{Compliance with Ethical Standards}

Conflict of interest All authors claim that there are no conflicts of interest.

Open Access This article is distributed under the terms of the Creative Commons Attribution 4.0 International License (http:// creativecommons.org/licenses/by/4.0/), which permits unrestricted use, distribution, and reproduction in any medium, provided you give appropriate credit to the original author(s) and the source, provide a link to the Creative Commons license, and indicate if changes were made.

\section{References}

1. Cohen JD, Perlstein WM, Braver TS, Nystrom LE, Noll DC, Jonides J, et al. Temporal dynamics of brain activation during a working memory task. Nature 1997, 386: 604-608.

2. Edin F, Klingberg T, Johansson P, McNab F, Tegner J, Compte A. Mechanism for top-down control of working memory capacity. Proc Natl Acad Sci U S A 2009, 106: 6802-6807.

3. Hare TA, Camerer CF, Rangel A. Self-control in decisionmaking involves modulation of the vmPFC valuation system. Science 2009, 324: 646-648.

4. Mitchell DG, Luo Q, Avny SB, Kasprzycki T, Gupta K, Chen G, et al. Adapting to dynamic stimulus-response values: differential contributions of inferior frontal, dorsomedial, and dorsolateral regions of prefrontal cortex to decision making. J Neurosci 2009, 29: 10827-10834.

5. Bi T, Fang F. Impaired face perception in individuals with autism spectrum disorder: Insights on diagnosis and treatment. Neurosci Bull 2017, 33: 757-759.

6. Hu ML, Zong XF, Mann JJ, Zheng JJ, Liao YH, Li ZC, et al. A review of the functional and anatomical default mode network in schizophrenia. Neurosci Bull 2017, 33: 73-84.

7. Herrington JD, Mohanty A, Koven NS, Fisher JE, Stewart JL, Banich MT, et al. Emotion-modulated performance and activity in left dorsolateral prefrontal cortex. Emotion 2005, 5: 200-207.

8. Perlstein WM, Elbert T, Stenger VA. Dissociation in human prefrontal cortex of affective influences on working memoryrelated activity. Proc Natl Acad Sci U S A 2002, 99: 1736-1741.

9. Godinho F, Magnin M, Frot M, Perchet C, Garcia-Larrea L. Emotional modulation of pain: is it the sensation or what we recall? J Neurosci 2006, 26: 11454-11461.

10. Pavuluri MN, Passarotti AM, Harral EM, Sweeney JA. An fMRI study of the neural correlates of incidental versus directed emotion processing in pediatric bipolar disorder. J Am Acad Child Adolesc Psychiatry 2009, 48: 308-319.

11. Sergerie K, Lepage M, Armony JL. A face to remember: emotional expression modulates prefrontal activity during memory formation. Neuroimage 2005, 24: 580-585. 
12. Wager TD, Phan KL, Liberzon I, Taylor SF. Valence, gender, and lateralization of functional brain anatomy in emotion: a metaanalysis of findings from neuroimaging. Neuroimage 2003, 19 : 513-531.

13. Borod JC, Cicero BA, Obler LK, Welkowitz J, Erhan HM, Santschi C, et al. Right hemisphere emotional perception: evidence across multiple channels. Neuropsychology 1998, 12: 446-458.

14. Dolcos F, Diaz-Granados P, Wang L, McCarthy G. Opposing influences of emotional and non-emotional distracters upon sustained prefrontal cortex activity during a delayed-response working memory task. Neuropsychologia 2008, 46: 326-335.

15. Fales CL, Barch DM, Rundle MM, Mintun MA, Mathews J, Snyder AZ, et al. Antidepressant treatment normalizes hypoactivity in dorsolateral prefrontal cortex during emotional interference processing in major depression. J Affect Disord 2009, 112: 206-211.

16. Erk S, Mikschl A, Stier S, Ciaramidaro A, Gapp V, Weber B, et al. Acute and sustained effects of cognitive emotion regulation in major depression. J Neurosci 2010, 30: 15726-15734.

17. Murphy DN, Boggio P, Fregni F. Transcranial direct current stimulation as a therapeutic tool for the treatment of major depression: insights from past and recent clinical studies. Curr Opin Psychiatry 2009, 22: 306-311.

18. Nitsche MA, Boggio PS, Fregni F, Pascual-Leone A. Treatment of depression with transcranial direct current stimulation (tDCS): a review. Exp Neurol 2009, 219: 14-19.

19. Schneider HD, Hopp JP. The use of the Bilingual Aphasia Test for assessment and transcranial direct current stimulation to modulate language acquisition in minimally verbal children with autism. Clin Linguist Phon 2011, 25: 640-654.

20. Kincses TZ, Antal A, Nitsche MA, Bartfai O, Paulus W. Facilitation of probabilistic classification learning by transcranial direct current stimulation of the prefrontal cortex in the human. Neuropsychologia 2004, 42: 113-117.

21. Marshall L, Molle M, Hallschmid M, Born J. Transcranial direct current stimulation during sleep improves declarative memory. J Neurosci 2004, 24: 9985-9992.

22. Fregni F, Boggio PS, Nitsche M, Bermpohl F, Antal A, Feredoes $\mathrm{E}$, et al. Anodal transcranial direct current stimulation of prefrontal cortex enhances working memory. Exp Brain Res 2005, 166: 23-30.

23. Beeli G, Koeneke S, Gasser K, Jancke L. Brain stimulation modulates driving behavior. Behav Brain Funct 2008, 4: 34.

24. Boggio PS, Campanha C, Valasek CA, Fecteau S, Pascual-Leone A, Fregni F. Modulation of decision-making in a gambling task in older adults with transcranial direct current stimulation. Eur $\mathrm{J}$ Neurosci 2010, 31: 593-597.

25. Hecht D, Walsh V, Lavidor M. Transcranial direct current stimulation facilitates decision making in a probabilistic guessing task. J Neurosci 2010, 30: 4241-4245.

26. Fecteau S, Knoch D, Fregni F, Sultani N, Boggio P, PascualLeone A. Diminishing risk-taking behavior by modulating activity in the prefrontal cortex: a direct current stimulation study. J Neurosci 2007, 27: 12500-12505.

27. Penolazzi B, Di Domenico A, Marzoli D, Mammarella N, Fairfield B, Franciotti R, et al. Effects of transcranial direct current stimulation on episodic memory related to emotional visual stimuli. PLoS One 2010, 5: e10623.

28. Liebetanz D, Fregni F, Monte-Silva KK, Oliveira MB, Amanciodos-Santos A, Nitsche MA, et al. After-effects of transcranial direct current stimulation (tDCS) on cortical spreading depression. Neurosci Lett 2006, 398: 85-90.

29. Rigonatti SP, Boggio PS, Myczkowski ML, Otta E, Ribeiro RB, Nitsche MA, et al. Transcranial direct stimulation and fluoxetine for the treatment of depression. Eur Psychiatry 2008, 23: 74-76.

30. DaSilva AF, Volz MS, Bikson M, Fregni F. Electrode positioning and montage in transcranial direct current stimulation. J Vis Exp 2011.

31. Nitsche MA, Cohen LG, Wassermann EM, Priori A, Lang N, Antal A, et al. Transcranial direct current stimulation: State of the art 2008. Brain Stimul 2008, 1: 206-223.

32. Boggio PS, Zaghi S, Fregni F. Modulation of emotions associated with images of human pain using anodal transcranial direct current stimulation (tDCS). Neuropsychologia 2009, 47: 212-217.

33. Indersmitten T, Gur RC. Emotion processing in chimeric faces: hemispheric asymmetries in expression and recognition of emotions. J Neurosci 2003, 23: 3820-3825.

34. Adolphs R, Jansari A, Tranel D. Hemispheric perception of emotional valence from facial expressions. Neuropsychology 2001, 15: 516-524.

35. Fertonani A, Rosini S, Cotelli M, Rossini PM, Miniussi C. Naming facilitation induced by transcranial direct current stimulation. Behav Brain Res 2010, 208: 311-318.

36. Ferrucci R, Mameli F, Guidi I, Mrakic-Sposta S, Vergari M, Marceglia $\mathrm{S}$, et al. Transcranial direct current stimulation improves recognition memory in Alzheimer disease. Neurology 2008, 71: 493-498.

37. Ohn SH, Park CI, Yoo WK, Ko MH, Choi KP, Kim GM, et al. Time-dependent effect of transcranial direct current stimulation on the enhancement of working memory. Neuroreport 2008, 19: $43-47$.

38. Andrews SC, Hoy KE, Enticott PG, Daskalakis ZJ, Fitzgerald PB. Improving working memory: the effect of combining cognitive activity and anodal transcranial direct current stimulation to the left dorsolateral prefrontal cortex. Brain Stimul 2011, 4: 84-89.

39. Jolij J, Lamme VAF. Repression of unconscious information by conscious processing: Evidence from affective blindsight induced by transcranial magnetic stimulation. Proc Natl Acad Sci USA 2005, 102: 10747-10751.

40. Sinnett S, Snyder JJ, Kingstone A. Role of the lateral prefrontal cortex in visual object-based selective attention. Exp Brain Res 2009, 194: 191-196.

41. Buschman TJ, Miller EK. Serial, covert shifts of attention during visual search are reflected by the frontal eye fields and correlated with population oscillations. Neuron 2009, 63: 386-396.

42. Antal A, Kincses TZ, Nitsche MA, Bartfai O, Paulus W. Excitability changes induced in the human primary visual cortex by transcranial direct current stimulation: direct electrophysiological evidence. Invest Ophthalmol Vis Sci 2004, 45: 702-707.

43. Beer JS, John OP, Scabini D, Knight RT. Orbitofrontal cortex and social behavior: integrating self-monitoring and emotion-cognition interactions. J Cogn Neurosci 2006, 18: 871-879.

44. Russell M, Goodman T, Wang Q, Groshong B, Lyeth BG. Gender differences in current received during transcranial electrical stimulation. Front Psychiatry 2014, 5: 104.

45. Boggio PS, Zaghi S, Villani AB, Fecteau S, Pascual-Leone A, Fregni F. Modulation of risk-taking in marijuana users by transcranial direct current stimulation (tDCS) of the dorsolateral prefrontal cortex (DLPFC). Drug Alcohol Depend 2010, 112: $220-225$. 